\title{
Editorial: Is Heterozygosity for a Wilson's Disease Gene Defect an Important Underlying Cause of Infantile and Childhood Copper Toxicosis Syndromes?
}

\author{
George J. Brewer*
}

Departments of Human Genetics and Internal Medicine, University of Michigan, Ann Arbor, Michigan

In this editorial, I would like to provide basic information on two seemingly disparate topics, and then bring them together in a unifying hypothesis that claims one is an underlying cause of the other. The two topics are first, the heterozygous state for a Wilson's disease genetic defect, and second, the occurrence of infantile and early childhood cirrhosis due to copper toxicity. I hypothesize that the latter primarily occurs in the presence of heterozygosity for Wilson's disease, and that this heterozygosity is often an important causal factor.

By way of background, Wilson's disease is an inherited disorder of copper metabolism in which there is a failure in biliary excretion of excess copper [1,2]. As a result, these patients are not able to eliminate the small excess of copper that is present in most human diets. This daily accumulation leads to the liver becoming excessively loaded with copper, and it is damaged early in childhood. At some point, patients may present with clinically obvious liver disease, while in others, the liver disease may remain subclinical. As the liver is increasingly unable to store the excess copper, levels build up elsewhere, and the brain is the next most sensitive organ. These patients develop neurologic symptoms of a movement disorder type and often psychiatric symptomatology as well. Fortunately, there are now good treatments for Wilson's disease, and if these patients are diagnosed and treated early enough, recovery is usually substantial, they can live a normal or near-normal life, and have a normal or near-normal life expectancy [1-4]. The disease is rather rare, with frequencies around the world estimated at about 1 in 40,000. This case frequency estimate leads to an estimated heterozygous carrier frequency of $1 \%$.

The gene responsible for Wilson's disease has been cloned [5-7]. It is called ATP7B and codes for a protein which has copper- and membrane-binding specificities, and ATPase activity. This protein is responsible in some manner for helping the

Contract grant sponsor: FDA; Contract grant number: FD-R-000179; Contract grant sponsor: General Clinical Research Center of the University of Michigan Hospitals; Contract grant number: M01-RR00042.

*Correspondence to: George J. Brewer, M.D., The University of Michigan Medical School, Department of Human Genetics, 4708 Medical Science II, Ann Arbor, MI 48109-0618.

Received for publication 3 November 1999; Accepted 3 November 1999

(C) 2000 Wiley-Liss, Inc. 
liver to excrete copper into the bile. As with many human recessive genetic disorders, there are a very large number, over 100 so far, of identified mutations which either knock out or seriously cripple the Wilson's disease gene, and result in the clinical disease [8].

From the genetic perspective, Wilson's disease is viewed as a recessive disorder. By this it is meant, of course, that it takes two copies of the defective gene, either in homozygous form or compound heterozygous form, to produce the disease. However, over the years that we have been working on Wilson's disease and diagnosing affected patients and differentiating them from heterozygous carriers, it has become clear to us that the Wilson's disease gene is not completely recessive [1,9-11]. In the first place, whereas $80-90 \%$ of affected patients have very low to modestly reduced serum ceruloplasmin $(\mathrm{Cp})$ levels, 10-20\% of carriers also have modestly low levels of serum $\mathrm{Cp}[1,2]$. In keeping with the reduced serum Cp levels, heterozygotes also have an abnormal rate of incorporation of radio-copper into $\mathrm{Cp}$ for release into the blood. In this procedure, developed by others [12], radio-labeled copper is administered either intravenously or orally at time zero. At 1-2 hr following oral administration (and sooner of course with IV administration), there is a blood peak of radioactivity. After that the level of blood radioactivity decreases sharply and then, over the next $24 \mathrm{hr}$, slowly builds up again as radio-copper is incorporated into $\mathrm{Cp}$ in the liver and the $\mathrm{Cp}$ is released into the blood. Thus, there is a second radioactive peak at $24 \mathrm{hr}$. In Wilson's disease patients, as is clearly shown in Figure 1 taken from our work [1], there is a markedly reduced rate of release of radio-labeled Cp into the blood compared to normals. The main point to be made here is that heterozygous carriers have an intermediate overall rate of appearance of radio-copper, and in some cases the abnormality is as extreme as it is in affected patients (Fig. 1).

Heterozygous carriers also accumulate copper abnormally in their livers (Fig. 2).

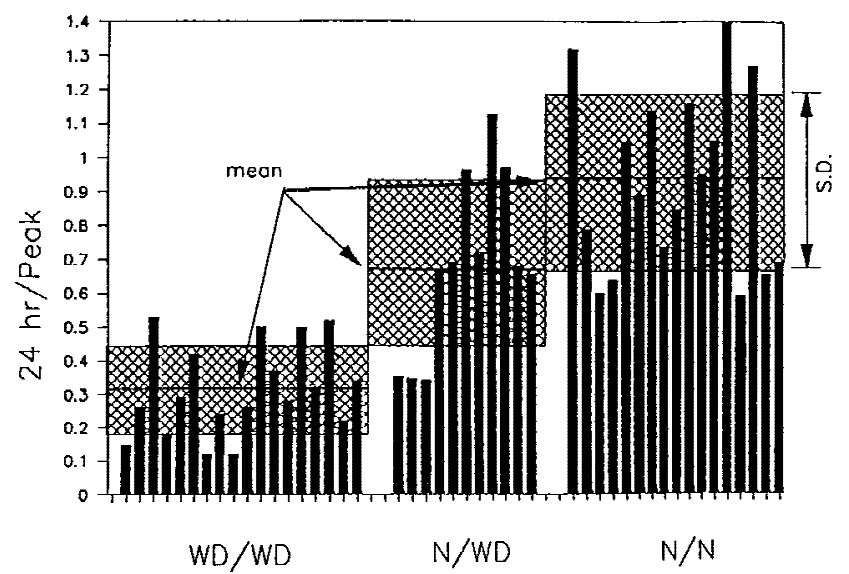

Fig. 1. Incorporation of orally administered ${ }^{64}$ copper into ceruloplasmin at $24 \mathrm{hr}$. Means and standard deviations are displayed. The vertical scale shows the ratio of the 24 -hr value to the peak at 1 or $2 \mathrm{hr}$. $\mathrm{WD} / \mathrm{WD}=$ homozygous affected, N/WD = heterozygous, N/N = homozygous normal. Hatched portion $=$ mean $+1 \mathrm{SD}$. These data show that heterozygous carriers of the Wilson's disease gene have intermediate values between normal and affected individuals, and often are as abnormal as affected patients. Figure reproduced with permission from Brewer and Yuzbasiyan-Gurkan [1]. 
In working up siblings of affected patients in order to differentiate affected patients from carriers, we have often had to do liver biopsies for final diagnosis. Two-thirds of the siblings who do not have Wilson's disease will be heterozygous carriers on probablistic grounds. We find an elevated hepatic copper in the majority of siblings who are likely heterozygotes (Fig. 2, lower panel). Figure 2 (upper panel) also shows data on a number of nonsibling patients we worked up for Wilson's disease and excluded the diagnosis. Chances are good that many of these subjects are heterozygous carriers, and many have abnormally elevated hepatic copper levels.

We have much more extensive data on urine copper values in siblings of affected patients. Again, after we exclude the diagnosis of Wilson's disease, two-thirds of the remaining siblings will be heterozygotes, based on probabilities. As can be seen from Figure 3, urine copper levels are elevated abnormally in a little over half of the calculated number of heterozygous siblings.

Thus, summarizing the data in heterozygous carriers of the Wilson's disease gene defect, we find that the gene really is codominant in many respects, with intermediate abnormalities of ceruloplasmin release into the blood and copper accumulation abnormalities in liver and urine. These data suggest that under environmental circumstances of increased copper ingestion, Wilson's disease could be made into a dominant disorder, that is, copper toxicity could be seen in heterozygous carriers.

In examining the data from some of the infantile and childhood cirrhosis syndromes associated with high levels of copper in the liver, it has occurred to me that those infants and small children may have exhibited precisely what is hypothesized above, i.e., that the affected children are heterozygous carriers of the Wilson's disease gene defect.

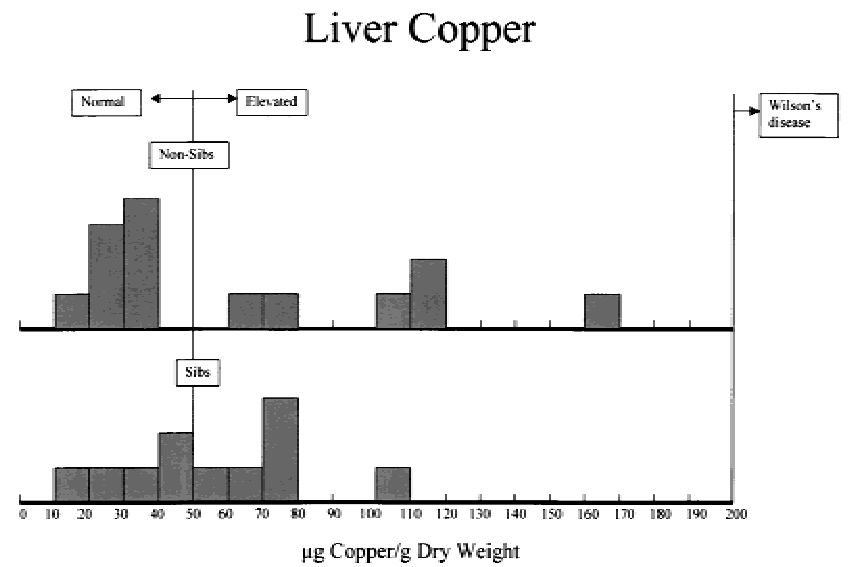

Fig. 2. Elevated hepatic copper levels in presumptive heterozygotes for the Wilson's disease gene. The first vertical line (at $50 \mu \mathrm{g}$ ) shows the upper limit of normal. The second vertical line (at $200 \mu \mathrm{g}$ ) shows the lower limit at which the diagnosis of Wilson's disease can be made. The lower panel shows data in siblings of affected patients, $2 / 3$ of whom are heterozygotes, on a probabalistic basis. Since $n=11,7$ or 8 heterozygotes would be expected. Elevated hepatic copper levels are seen in 6 individuals, indicating that $3 / 4$ or more of heterozygotes have elevated hepatic copper levels. The upper panel shows hepatic copper values in other individuals suspected of having Wilson's disease. None of these 15 persons had Wilson's disease, but hepatic copper levels were elevated in 6 . These 6 individuals are very likely heterozygous for the Wilson's gene defect. 
Frequency Distribution of 24-hour Urine Copper Values for 206 non-Wilsonian Siblings of Wilson's Disease Patients

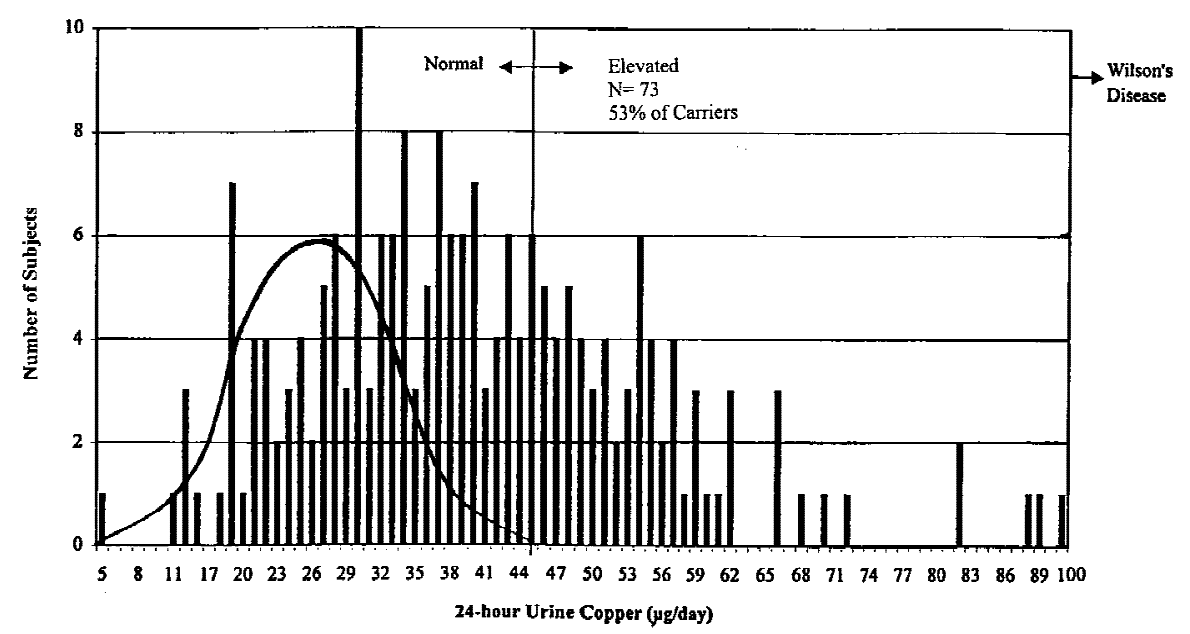

Fig. 3. Distribution of urine copper values in 206 siblings of diagnosed Wilson's disease patients. The diagnosis of Wilson's disease was excluded in these 206 siblings. Probabilistically, 2/3 of these siblings, or 138 of them, are carriers of the Wilson's disease gene. 73, or 53\% of the estimated 138 carriers, have elevated urine copper values. The theoretical curve for urine copper values in normal individuals is superimposed on the left hand side of the distribution. A portion of these data have been previously published [10].

To review these syndromes briefly, between 1900 and 1980, 138 infants and young children died in the Tyrolean area of Western Austria from liver cirrhosis associated with very high levels of hepatic copper [13]. Since about 1980, this syndrome has essentially disappeared. A common feature of this disorder was the feeding of infants and young children with a preparation called "feeding milk," which is a 1:1 mixture of unpasturized cow's milk with water. This material was heated for about $20 \mathrm{~min}$ in copper or brass pots. When this process was repeated later, investigators found that a very high level of copper from the pots appeared in the feeding milk [13]. Traditional copper cooking utensils were gradually replaced with modern industrial vessels, and this disease gradually disappeared by about 1980 .

Assuming that the frequency of carriers of the Wilson's disease gene defect is about $1 \%$ in the Tyrolean area as it is currently estimated to be in most populations, the data on the number of infants affected with Tyrolean infantile cirrhosis are quite compatible with the hypothesis that the affected children were carriers of the Wilson's disease gene. The population size involved was about 45,000 [13]. At its peak, the epidemic had about 3.8 affected infant cases per year [13]. That number of Wilson's disease carriers would easily have been generated from a population of this size. The Tyrolean disease appeared to be genetically determined, as it occurred in some families but not in others in the same environment and presumably using the same feeding milk preparation, and was recessive, as in many families multiple siblings were involved [13].

Indian childhood cirrhosis is remarkable similar to Tyrolean infantile cirrhosis [14-19]. It occurs in India in infants or very young children fed milk stored in brass 
or copper containers. The milk has been shown to have a very high copper content, and the disease is familial, with a recessive segregation pattern. Again, the cirrhosis appears to be due to copper toxicity in the liver. The role of copper in this case is supported not only by the high copper concentrations in the liver but also by rapid improvement with penicillamine treatment.

The third syndrome is referred to as idiopathic copper toxicosis [20]. This syndrome has occurred in many countries, and often other names are used, such as copper-associated childhood cirrhosis, Indian childhood cirrhosis-like, and copperassociated liver disease in childhood. In a review by Muller et al. [20], 15 previous reports were analyzed. Many of the cases appeared in infants or very young children, but some occurred in children up to 10 years of age. A feature in common was cirrhosis associated with very high levels of hepatic copper. In many, but not in all cases, increased ingestion of copper had been shown. There was often involvement of more than one sibling. The majority of the cases of ICT appear associated with elevated copper in drinking water. For example, in the review of Muller et al. [20], there were 15 papers reporting ICT. In eight of these, copper in tap water was reported, and in five of the eight (involving eight patients), copper levels were elevated in drinking water. In the other three reports (involving six patients), presumably the copper came from some source other than the drinking water. Involvement of a genetic (or at least a familial) component was suggested in some cases by involvement of multiple siblings.

In summary then, the infantile and childhood cirrhosis syndromes associated with elevated levels of hepatic copper all have in common that they occur in infants and young children, that an increased intake of copper can usually be identified, that copper toxicity appears to be occurring in the liver causing cirrhosis, and that there are indications of a genetic contribution segregating in a recessive manner.

Given that we have shown that heterozygous carriers of the Wilson's disease gene are susceptible to copper accumulation, even in an environment of presumed normal copper intake, it is a reasonable hypothesis that these infantile and childhood copper toxicosis/cirrhosis syndromes are often, if not always, a result of disease in heterozygous carriers of the Wilson's disease gene defect. Under these circumstances, the disease would have been converted to a dominant by the environmental situation of a high copper intake. Unlike most dominant genetic defects, the disease is not seen in a parent because the heterozygous parent is not exposed to the same environment as the heterozygous infant, that is, the environment of a high copper intake. The infants and young children are either drinking milk with high copper or taking formula made with copper-contaminated drinking water.

Can this hypothesis be tested? To the extent that parents of children who were affected with this syndrome are still available, either in India or Tyrol, or in some of the ICT cases, one of the two parents in these families should be heterozygous for a Wilson's disease gene defect. It seems to me that perhaps the easiest way to test the hypothesis would be to set up a DNA test for the most common one or two mutations producing Wilson's disease, and to study these parents to determine the frequency of those mutations. For example, if mutations which normally comprise about $50 \%$ of Wilson's disease mutations in a given population are tested for, it would be expected that one out of every two families would have a parent with one of these mutations, if a large proportion of the cases involve Wilson's disease heterozygosity. 


\section{REFERENCES}

1. Brewer GJ, Yuzbasiyan-Gurkan V. Wilson disease. Medicine 1992;71:139-164.

2. Scheinberg IH, Sternleib I. Wilson's disease. In: Smith LH, Jr, editor. Major problems in internal medicine. Philadelphia: WB Saunders Company; 1984. Vol 23.

3. Brewer GJ, Dick RD, Johnson VD, Brunberg JA, Kluin KJ, Fink JK. Treatment of Wilson's disease with zinc: XV Long-term follow-up studies. J Lab Clin Med 1998;132:264-278.

4. Brewer GJ, Johnson VD, Dick RD, Kluin KJ, Fink JK, Brunberg JA. Treatment of Wilson disease with ammonium tetrathiomolybdate II. Initial therapy in 33 neurologically affected patients and follow-up with zinc therapy. Arch Neurol 1996;53:1017-1025.

5. Bull PC, Thomas GR, Rommens JM, Forbes JR, Cox DW. The Wilson disease gene is a putative copper transporting P-type ATPase similar to the Menkes gene. Nat Genet 1993;5(4):327-337.

6. Tanzi RE, Petrukhin K, Chernov I, Pellequer JL, Wasco W, Ross B, et al. The Wilson disease gene is a copper transporting ATPase with homology to the Menkes disease gene. Nat Genet 1993;5(4): 44-50.

7. Yamaguchi Y, Heiny ME, Gitlin JD. Isolation and characterization of a human liver cDNA as a candidate gene for Wilson disease. Biochem Biophys Res Commun 1993;197:271-277.

8. Roberts EA, Cox DW. Wilson disease. Bailliere's Clinical Gastroenterology 1998;12:237-256.

9. Brewer GJ, Hill GM, Prasad AS, Cossack ZT, Rabbani P. Oral zinc therapy for Wilson's disease. Ann Intern Med 1983;99:314-320.

10. Yuzbasiyan-Gurkan V, Johnson V, Brewer GJ. Diagnosis and characterizations of presymptomatic patients with Wilson's disease and the use of molecular genetics to aid in the diagnosis. J Lab Clin Med 1991;118:458-465.

11. Brewer GJ, Dick RD, Yuzbasiyan-Gurkan V, Johnson V, Wang Y. Treatment of Wilson's disease with zinc: XIII. Therapy with zinc in presymptomatic patients from the time of diagnosis. J Lab Clin Med 1997;129:649-652.

12. Sternlieb I, Morell AG, Bauer CD, Combes B, DeBobes-Sternberg S, Scheinberg IH. Detection of the heterozygous carrier of the Wilson's disease gene. J Clin Invest 1960;40:707-715.

13. Müller T, Feichtinger H, Berger H, Müller W. Endemic tyrolean infantile cirrhosis: An exogenetic disorder. Lancet 1996;347:877-880.

14. Tanner MS, Portman B, Mowat AP, Williams R, Pandit AN, Mills CF, Bremner I. Increased hepatic copper concentration in Indian childhood cirrhosis. Lancet 1979;1(8128):1203-1205.

15. Popper H, Goldfischer S, Sternlieb, Nayak NC, Madhavan TV. Cytoplasmic copper and its toxic effects. Studies in Indian childhood cirrhosis. Lancet 1979;1(8128):1205-1208.

16. Tanner MS, Kantarjian AH, Bhave SA, Pandit AN. Early introduction of copper-contaminated animal milk feeds as a possible cause of Indian childhood cirrhosis. Lancet 1983;2(8357):992-995.

17. Bhave SA, Pandit AN, Pradhan AM, Sidhaye DG, Kantarjian A, Williams A, Talbot IC, Tanner MS Liver disease in India. Arch Dis Child 1982;57:922-928.

18. Pandit A, Bhave S. Present interpretation of the role of copper in Indian childhood cirrhosis. Am J Clin Nutr 1996;63:830S-835S.

19. Bavdekar AR, Bhave SA, Pradhan AM, Pandit AN, Tanner MS. Long term survival in Indian childhood cirrhosis treated with D-penicillamine. Arch Dis Child 1996;74:32-35.

20. Müller T, Müller W, Freichtinger H. Idiopathic copper toxicosis. Am J Clin Nutr 1998;67(Suppl): 1082S-1086S 